\title{
Remorquage el immersion de la plate-forme ELF TP 1
}

\section{Lucien Pliskin}

Directeur Technique à Sainrapt et Brice

Conseiller Technique Sea Tank Co

La plateforme pétrolière ELF TP 1 a été mise en place en juin 1976, sur le Champ de Frigg, en Mer du Nord, pour le compte d'ELF Norge. Ce Champ est situé à mi-distance des côtes anglaise et norvégienne par environ $60^{\circ}$ de latitude Nord.

La plateforme TP 1 est une plateforme poids en béton. Comme toute plate-forme, elle doit permettre la



Figure 1 - Remorquage de la plateforme TP1 création d'une usine en haute mer, en l'occurence une station de traitement du gaz produit par le Champ de Frigg.

Elle a été construite par Sea Tank Co/Mac Alpine à Ardyne Point, dans le Loch Striven, près de Glasgow.

Commencée dans une souille de $10 \mathrm{~m}$ de profondeur, sa construction s'est poursuivie en flottant, à $200 \mathrm{~m}$ du rivage, jusqu'à l'achèvement de la structure en béton.

Un premier remorquage de 30 milles nautiques a conduit la plateforme dans le Loch Fyne afin de permettre une immersion profonde et faciliter ainsi la mise en place du pont supérieur.

La plateforme a été ensuite remorquée sur 800 milles nautiques, jusqu'au Champ de Frigg, où elle a été immergée et posée sur le fond de la mer. (Fig. 1)

Avant de décrire ces opérations marines, je rappellerai rapidement ce qu'est la plateforme poids TP 1 .

\section{Les plateformes poids}

Les plateformes poids présentent la particularité d'être dimensionnées de manière à ce que, posées sur le fond marin, elles résistent aux effets conjugués de la houle, du courant et du vent par leur propre poids, sans nécessiter d'ancrages.

Les plateformes Sea Tank Co sont constituées par un caisson de base, formant fondation, à partir duquel s'élèvent des colonnes supportant un pont de travail métallique.

Cette conception présente l'intérêt de minimiser les efforts de la houle sur la structure. Ses effets se manifestent, en fait, avec le maximum d'ampleur près de la surface, donc sur les colonnes qui n'offrent qu'un maittre couple réduit. 


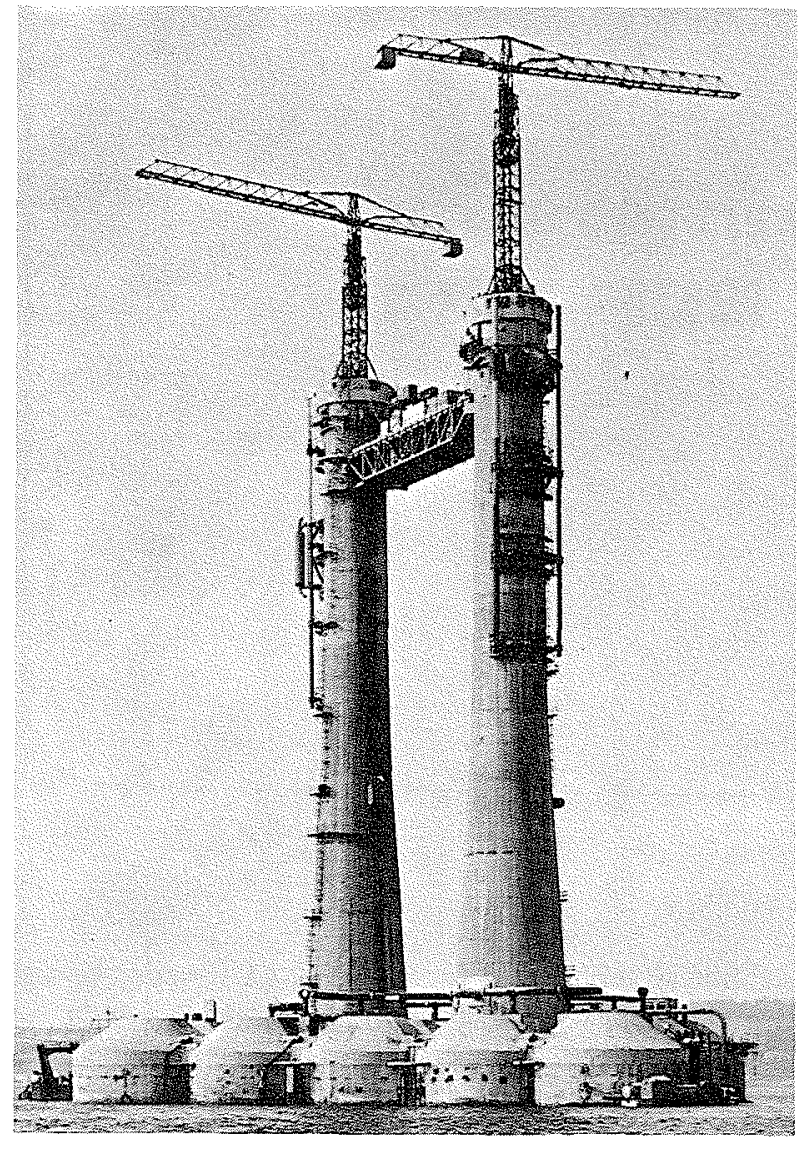

Photo 1 - Plateforme TP1 en fin de construction

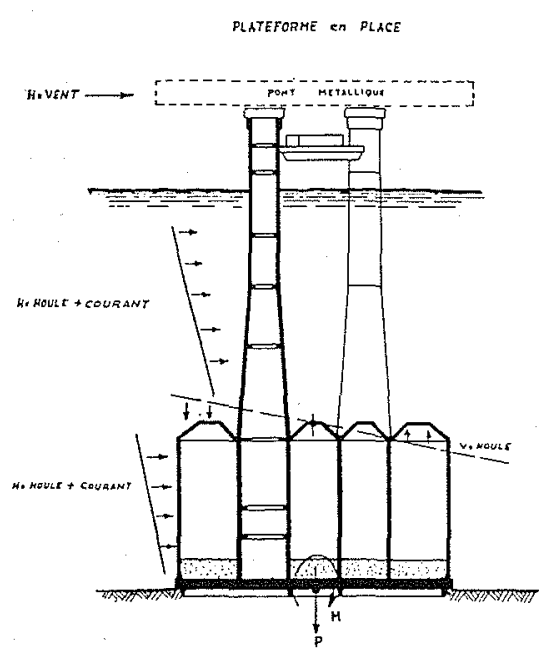

Figure 2 - Efforts agissant sur la plateforme TP1

Les efforts à équilibrer n'en restent pas moins extrêmement importants. Pour éviter le renversement ou le glissement de la plateforme, il convient de lui conférer un poids suffisant. (fig. 2)

C'est pourquoi le caisson et les colonnes sont réalisés en béton, matériau qui a, de plus, l'avantage d'être à peu près insensible à toute corrosion marine.

Appliqué au cas particulier de TP 1 , ce concept conduit à une structure comportant un caisson de $72 \mathrm{~m} \times 72 \mathrm{~m}$ et de $44 \mathrm{~m}$ de hauteur dans lequel s'encastrent deux colonnes de $80 \mathrm{~m}$ de hauteur dont le diamètre varie de $13 \mathrm{~m}$ à $8 \mathrm{~m}$ près de la surface de la mer. Le pont a une longueur de $90 \mathrm{~m}$ et une largeur de 44 m environ. (fig. 3 )

La plateforme est destinée à être posée sur des fonds sableux par $104 \mathrm{~m}$ de profondeur.

Elle doit pouvoir résister aux efforts de la houle dite centenaire, caractérisée par un creux de $29 \mathrm{~m}$ et une période de 15 secondes.

Cette houle engendre sur la structure des forces hydrodynamiques qui s'élèvent à $30000 \mathrm{t}$ pour l'effort horizontal, à $\pm 20000 \mathrm{t}$ pour l'effort vertical, et à un moment de renversement d'environ $700000 \mathrm{tm}$.

La construction de la plateforme a nécessité $50000 \mathrm{~m}^{3}$ de béton de structure et $15000 \mathrm{~m}^{3}$ de béton de ballast.

Son déplacement est de $166000 \mathrm{t}$ en lège et de $214000 \mathrm{t}$ en immersion avec un tirant d'eau de $65 \mathrm{~m}$. Lors du remorquage, le poids des charges placées en tête des colonnes est de $4000 \mathrm{t}$.

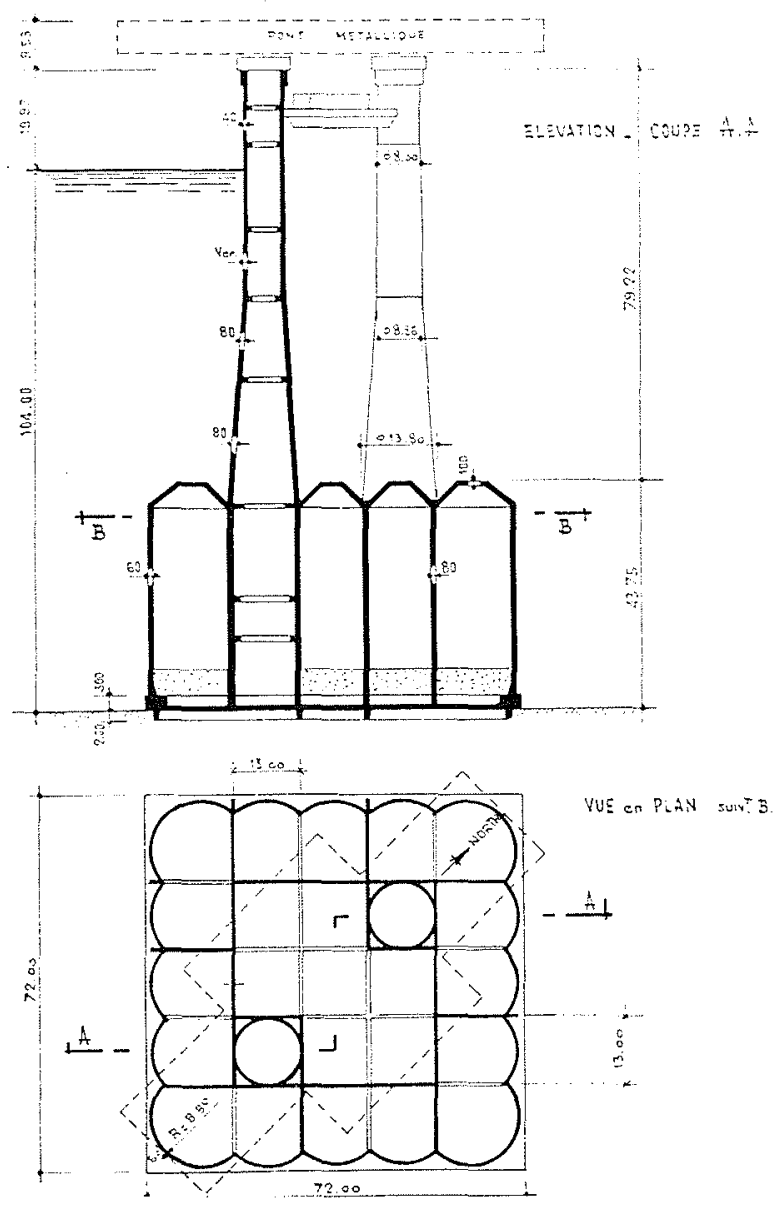

Figure 3 - Elévation et coupe 


\section{Essais en bassin et calculs hydrodynamiques}

Le comportement de la plateforme vis-à-vis de l'environnement marin a été vérifié tant par le calcul que par des essais en bassin de houle. Ces essais, réalisés par le Bassin des Carènes de la Marine Nationale, par le Labo. ratoire National d'Hydraulique d'EDF et par le NSMB à Wageningen ont porté sur différents points tels que :

- la vérification de la valeur des efforts exercés par des houles régulières et irrégulières sur la plateforme;

- l'effet d'écran créé par la présence de deux colonnes, en fonction de leur entr'axe et de l'incidence de la houle ;

- les phénomènes se manifestant lors de la "prise d'immersion" de la plateforme, c'est-à-dire lors du passage du toit du caisson sous la surface de la mer, en fonction de la période et du creux de la houle;

- le comportement de la plateforme en cours de remorquage, en houle régulière et irrégulière, en situation lège ou ballastée avec, dans ce dernier cas, un tirant d'eau de $65 \mathrm{~m}$.

Les calculs ont remarquablement confirmé tous ces essais. C'est ainsi que les résultats obtenus au moyen du PGHM (Programme Général d'Hydrodynamique Marine) de Franlab ont corroboré relativement finement les essais de comportement en flottaison effectués en bassin.

L'expérience que nous avons tirée de l'étude de la plateforme TP 1 nous conduit à penser qu'il semble aujourd'hui parfaitement possible d'obtenir, par le seul calcul, des résultats fiables et sûrs, tant en ce qui concerne les efforts hydrodynamiques agissant sur une structure immergée qu'en ce qui touche aux mouvements affectant une plateforme en flottaison soumise à la houle.

\section{Caractéristiques marines de la plateforme}

La géométrie de la plateforme est telle que la hauteur métacentrique GM est minimum lors de la "prise d'im. mersion". Avec l'immersion du caisson, un terme très important de stabilité de forme disparaît puisque la structure se porte alors sur les colonnes. (fig. 4)

Dans ce cas, et compte tenu d'une charge en tête de colonnes de $4000 \mathrm{t}$, le $\mathrm{GM}$ atteint une valeur minimum d'environ $1,00 \mathrm{~m}$.

Par ailleurs, le calcul comme les essais en bassin montrent que les périodes propres de la plateforme sont les suivantes :

\begin{tabular}{|l|c|c|}
\cline { 2 - 3 } \multicolumn{1}{c|}{} & $\begin{array}{c}\text { Structure lège } \\
\text { Tirant d'eau }: 35 \mathrm{~m}\end{array}$ & $\begin{array}{c}\text { Structure ballastée } \\
\text { Tirant d'eau }: 65 \mathrm{~m}\end{array}$ \\
\hline Roulis, tangage & $22 \mathrm{sec}$. & $63 \mathrm{sec}$. \\
Pilonnement & $15 \mathrm{sec}$. & $110 \mathrm{sec}$. \\
\hline
\end{tabular}



Figure 4 - Hauteur métacentrique G.M.

Ces valeurs montrent l'appréciable effet d"'amollissement" créé par le ballastage du caisson. Les accélérations horizontales maxima se produisant en tête de colonne, au niveau du pont, sont alors d'environ 0,15 à $0,20 \mathrm{~m} / \mathrm{sec}^{2}$ en présence d'une houle de $29 \mathrm{~m} /$ 15 secondes.

Ces faibles valeurs montrent tout l'intérêt qu'il y a à effectuer le remorquage avec un tirant d'eau de lordre de $65 \mathrm{~m}$

\section{Equipements et instrumentation de contrôle}

Les opérations marines impliquent la possibilité d'immerger par ballastage et d'émerger par déballastage la plateforme. Ceci est réalisé au moyen d'un module qui comporte essentiellement 4 pompes centrifuges permettant un débit de $1500 \mathrm{~m}^{3} / \mathrm{h}$ sous une charge de $40 \mathrm{~m}$.

Le caisson de la plateforme, conçu de manière à y créer 9 compartiments indépendants, peut ainsi ètre rempli ou vidé d'eau de mer. Les tuyauteries placées dans le radier du caisson, permettent une amenée différentielle d'eau dans l'un quelconque de ces compartiments.

Ces opérations supposent aussi la mise en ceuvre d'une instrumentation de contrôle qui permet de connaître à tout moment, entre autres :

- le niveau de l'eau dans chacun des compartiments indépendants ;

- la gîte par mesure de l'inclinaison de la plateforme selon deux directions orthogonales;

- le pied de pilote par 4 echosondeurs disposés aux angles du radier de la plateforme. 


\section{Opérations marines}

Les opérations marines recouvrent les opérations de mise en place du pont métallique sur les colonnes, le remorquage proprement dit et la mise en place par immersion de la plateforme sur le Champ de Frigg (fig. 5).

\section{Opération Loch Fyne}

Après terminaison sur le site d'Ardyne, la plateforme est remorquée sur 30 milles nautiques jusqu'au Loch Fyne où les profondeurs atteignent $180 \mathrm{~m}$.
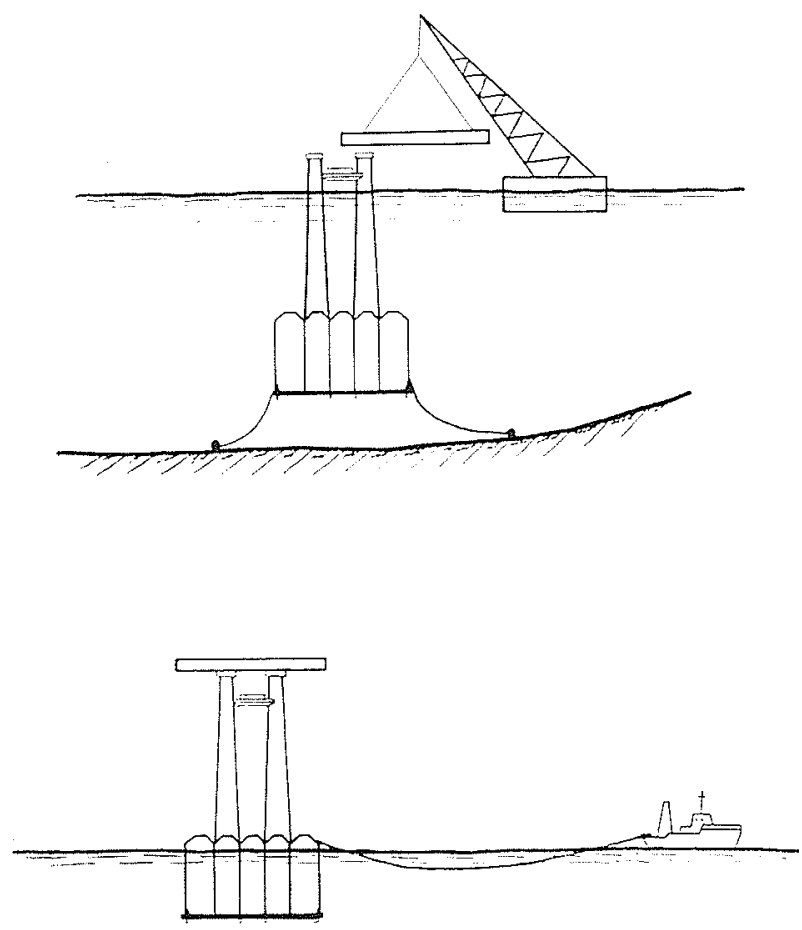

Figure 5 - Mise en place du pont

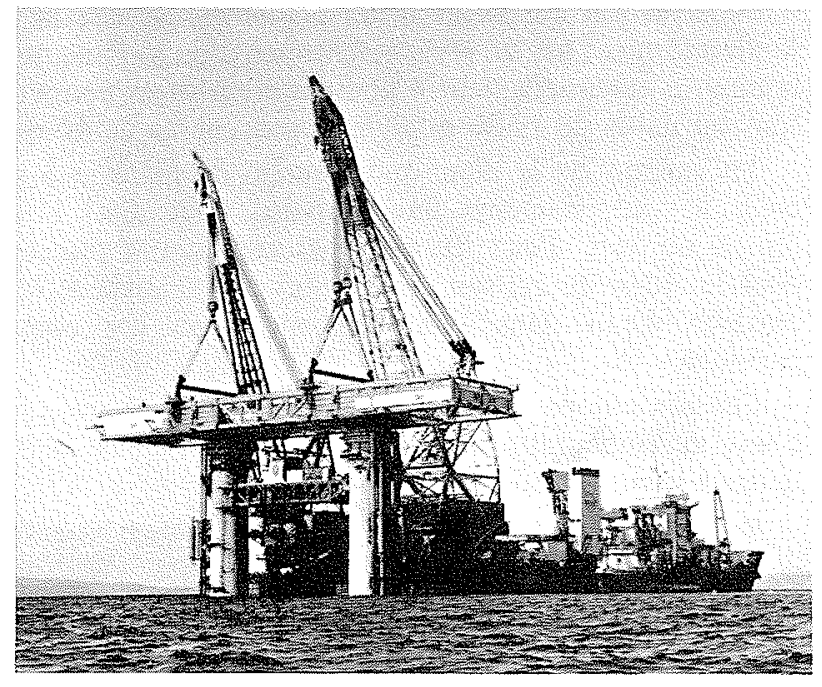

Photo 2 - Mise en place du pont
Dans ce Loch, il est procédé aux essais de tous les équipements et instruments de contrôle d'immersion.

La plateforme est alors immergée, jusqu'à présenter un tirant d'eau de $97 \mathrm{~m}$. Le pont métallique de $1800 \mathrm{t}$ est mis en place d'une pièce au moyen des deux barges ETPM 1601 et Meaders accouplées (photo 2).

Après solidarisation de ce pont de $94 \mathrm{~m}$ de longueur et de $20 \mathrm{~m}$ de largeur aux deux colonnes, la plateforme est partiellement déballastée. Des éléments complémentaires et des équipements sont mis en place sur le pont. La charge totale en tête de colonnes atteint alors $4000 \mathrm{t}$.

\section{Remorquage Loch Fyne -.. Frigg}

La plateforme terminée en Ecosse, il faut la remorquer jusqu'au Champ de Frigg. Ce remorquage doit s'effectuer dans une période comprise entre les mois de mai et de juillet, époque où les conditions de mer sont les plus favorables en Mer du Nord. Cette notion est d'ailleurs toute relative, puisqu'il est prévu de pouvoir rencontrer une tempête d'été définie par un creux de $17 \mathrm{~m}$ et une période de 12 secondes.

La présence de haut-fonds dans le North Channel, à la sortie de l'embouchure de la Clyde, conduit à réaliser la première partie du remorquage en lège (photo 3 ).

En haute mer, la plateforme est ballastée et remorquée avec un tirant d'eau de $65 \mathrm{~m}$.

Ce remorquage est effectué au moyen de 5 remorqueurs totalisant une puissance de $55000 \mathrm{CV}$ et exerçant un effort total au bollard de $435 \mathrm{t}$.

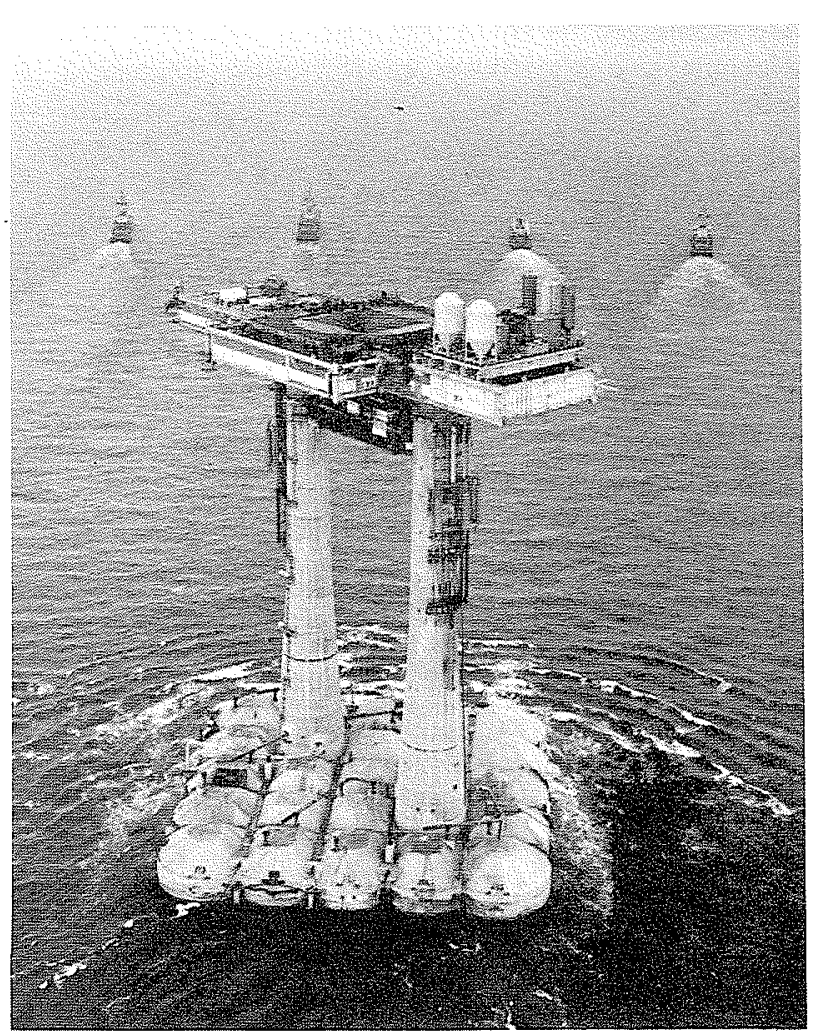

Photo 3 - Remorquage lège 
Le remorquage a commencé le 22 mai 1976 au soir, les prévisions météorologiques sur 48 heures étant satisfaisantes. Le 24 mai au soir, le convoi était sorti du North Channel. Il atteignait quelques heures après la ligne des $100 \mathrm{~m}$ de profondeur. La plateforme était alors ballastée de manière à atteindre le tirant d'eau de $65 \mathrm{~m}$, la vitesse de remorquage étant réduite à 1 nœud lors de la prise d'immersion.

Le 4 juin, 11 jours plus tard, le convoi se trouvait à 20 nautiques de Frigg. De mauvaises conditions de mer conduisaient à le ralentir, les opérations de mise en place ne pouvant se faire que par beau temps.

Ainsi, et malgré des mers de force 8 , la vitesse moyenne de remorquage en haute mer a atteint 2,8 nœuds.

Le comportement de la plateforme, durant ce voyage, a confirmé les résultats des essais et des calculs qui indiquaient une quasi-insensibilité de la structure à des états de mer centenaires.

\section{Mise en place de la plateforme sur le Champ de Frigg}

La mise en place impliquait une redistribution des remorqueurs, par rapport à la plateforme, pour permettre un contrôle aussi précis que possible de sa position. Cette reprise de nouveaux points d'amarrage ne pouvait se faire qu'en présence de bonnes conditions de mer (fig. 6).

Il s'est trouvé que le 4 juin au soir, les prévisions météorologiques sur 48 heures se sont révélées excellentes. Les opérations de redistribution des remorqueurs ont donc débuté vers minuit. Elles étaient achevées le 5 juin vers $10 \mathrm{~h}$. Entre temps, le ballastage de la plateforme avait repris de manière à se rapprocher du fond.

La mise en place proprement dite a commencé le 5 juin à $12 \mathrm{~h}$. A 15 h 30 ce même 5 juin, la plateforme reposait sur le fond marin et la ballastage complet du caisson était entrepris. Dès le lendemain matin, son poids étant suffisant, TP 1 pouvait résister à la tempête d'été.

Il convient de noter que cette mise en place constituait une opération délicate : il fallait en effet placer TP 1 à moins de $70 \mathrm{~m}$ d une plateforme métallique déjà en place.



Figure 6 - Positionnement à Frigg
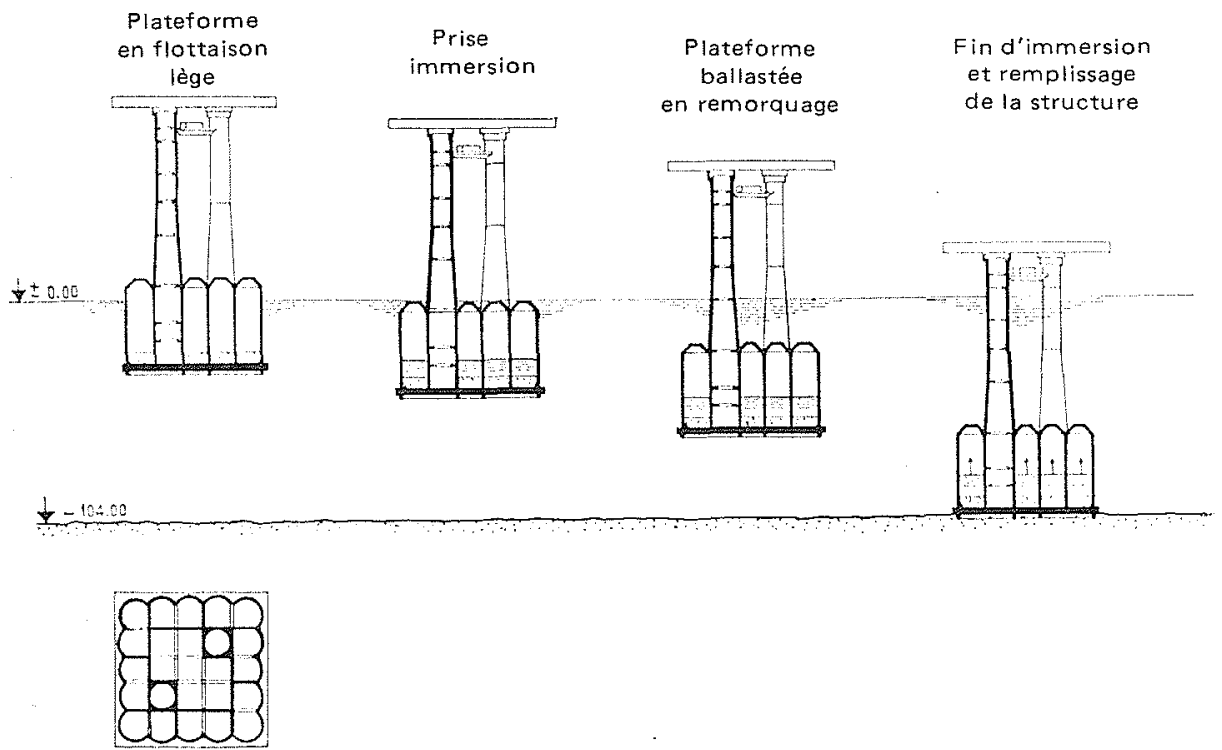

Figure 7 - Principe d'immersion 




Photo 4 - Vue du pont de la plateforme TP1 en place

Malgré ces difficultés, la précision de positionnement de TP 1 a été excellente puisque son axe se trouve à moins de $1 \mathrm{~m}$ du point théorique où il devait se situer tandis que son orientation a été respectée à moins de $0,5^{\circ}$ près.

\section{Conclusion}

Les conclusions qu'il semble possible de tirer de l'expérience des opérations de remorquage et d'immersion de la plateforme ELF TP 1 sont de trois ordres :

D'une part, l'intérêt que présentent les plateformes poids en béton est confirmé. L'exemple de cette plateforme Sea Tank Co montre tout l'avantage qu'offre ce type de structure qui permet de quitter le site de construction avec un pont de travail partiellement ou totalement équipé. Dans le cas de TP 1, la charge en tête n'était que de 4000 t. Cette charge peut être très largement majorée, si l'opérateur pétrolier le désire, moyennant une adaptation des dimensions de la plate- forme. C'est ainsi que Sea Tank Co dispose de projets permettant un remorquage en toute sécurité avec une charge en tête de $50000 \mathrm{t}$, c'est-à-dire avec un pont de travail totalement équipé et prêt à fonctionner.

Un autre avantage appréciable des plateformes poids tient dans la sécurité qu'elles permettent d'obtenir. En fait, la mise en place de TP 1 a duré moins de 4 heures, et 1 jour après le début de cette mise en place, cette plateforme était en mesure de résister aux effets d'une houle centenaire. Les prévisions météorologiques n'ayant encore qu'une faible amplitude, particulièrement en Mer du Nord, placer une plateforme en situation de sécurité en 24 heures ne constitue pas un point négligeable.

Enfin, et dans un autre ordre d'idées, il convient de souligner combien essais en bassin et calculs théoriques ont correctement prévu le comportement de la plateforme. Il est loisible de se demander si le temps n'est pas venu qui verra les constructeurs se fier de plus en plus aux seuls calculs, dans la mesure où ils procéderont à des extrapolations à l'intérieur d'une même famille de structures flottantes ou reposant sur le fond marin. 


\section{Discussion}

Président : M. VALLS

M. 1e Président remercie et félicite M. PLISKIN pour la clarté avec laquelle il a exposé le mémoire. Il souligne la souplesse du procédé de remorquage décrit et l'ingéniosité du système de ballastage. Il ouvre ensuite la discussion.

A-t-on procédé à une préparation du fond marin à l'emplacement où la plateforme devrait être échouée, demande M. SIREYJOL.

Aucune préparation du fond n'a été effectuée mais on a procédé à des reconnaissances topographiques en vue de s'assurer que le sol était raisonnablement plan ; afin de réaliser un bon contact entre le radier de la plateforme et les fonds marins, on a procédé à un remplissage des vides en utilisant les compartimentages entre bêches prévus, à cet effet, à la partie inférieure de l'ouvrage.

Dans une Communication présentée à la Session des 19 et 20 juin 1975 du Comité technique de la S.H.F. (Cf. La Houille Blanche $\left.n^{\circ} 7-8 / 1975\right)$, ajoute M. COUPRIE, j'ai eu l'occasion d'analyser les efforts fluctuants - du fait de la houle notamment- auxquels se trouve soumis le sol marin au voisinage et sous la fondation d'une plateforme. C'est un problème complexe d'hydrodynamique et de mécanique des sols qui met en jeu les pressions interstitielles et peu provoquer la "liquéfaction des sols".

Pour adapter la fondation au terrain, celle-ci est munie de "bêches" qui pénètrent plus ou moins profondément dans le sol. L'espace subsistant entre la base du caisson et le sol est rempli de matériaux de caractéristiques voisines de celles du sol. Des cheminées d'équilibrage mettent en communication le terrain de fondation et les couches d'eau quil le surmontent de façon que, lorsque les bêches poinçonnent le sol, il n'y ait pas, au sein de ce dernier, formation de surpressions susceptibles d'engendrer des "renards". Tout un corps de doctrine s'est développé sur ce sujet au cours des dernières années.
En terminant, M. COUPRIE pose la question suivante : A-t-on contrôlé la tension des remorques pendant le remorquage : en effet, si la plateforme est un semi-submersible peu sensible à la houle, les remorqueurs le sont ?

Ces efforts ont été contrôlés au début du remorquage et, en particulier, pendant la traversée du North Channel, répond M. PLISKIN ; mais, ces essais ont été effectués par la Marine britannique et nous ne disposons pas des résultats obtenus.

Quelle a été la durée totale de construction de la plateforme T.P. 1, demande M. DODU?

Ainsi que je l'ai dit, précise M. PLISKIN, la plateforme a été construite en Angleterre. Divers problèmes sociaux on retardé sa réalisation qui aurait dù être terminée en 18 mois à deux ans, y compris la préparation des souilles de construction. Aujourd'hui, ces souilles existent et une nouvelle plateforme pourrait être construite en 18 mois.

M. SOMMET (S.O.G.R.E.A.H.) soulève un intéressant problème : Lorsque, dit-il, au cours de l'immersion, la plateforme approche du fond, il peut se produire un effet de coin d'eau entre le fond et le sol, qui provoque un glissement latéral de la structure. Cet effet a-t-il été observé ?

Aucun effet de ce genre, répond M. PLISKIN, n'a été observé, ni au cours des essais sur modèle, ni en grandeur nature. Il n'aurait pu se produire, de toute façon, car nous avons tiré avantage, d'une part de la légère inclinaison du fond marin et, d'autre part, du fait que la vitesse d'échouage pouvait être contrôlée et limitée de façon très précise. Par ailleurs, les bêches délimitant les compartiments sous radier ont servi de premier ancrage lors de l'arrivée sur le fond, la plateforme ayant alors un poids apparent à peu près nul.

M. le Président clôt la discussion en remerciant, à nouveau, M. PLISKIN et donne la parole à M. MOGUILNY pour l'exposé de sa communication. 